\title{
On the Rate Distortion Functions of Memoryless Sources under a Magnitude-Error Criterion*
}

\author{
Hoi M. Ifegng and Stamatis Cambanis
}

Department of Statistics, University of North Carolina, Chapel Hill, North Carolina 27514

\begin{abstract}
We consider the evaluation of and bounds for the rate distortion functions of independent and identically distributed (i.i.d.) sources under a magnitudeerror criterion. By refining the ingeneous approach of Tan and Yao we evaluate explicitly the rate distortion functions of larger classes of i.i.d. sources and we obtain families of lower bounds for arbitrary i.i.d. sources.
\end{abstract}

\section{INTRODUCTION}

The rate distortion function of an independent and identically distributed (i.i.d.) source is clcarly equal to the rate distortion function of each random variable of the source. We evaluate the rate distortion function $R(D)$ of a random variable $X$ with density $p(x)$ satisfying certain conditions. A magnitude-error criterion is used throughout without further reminder.

The procedure used is based on the well known analytical expression of $R(D)$ and was introduced by Tan and Yao (1975), who calculated explicitly the rate distortion functions of an i.i.d. Gaussian source and of a certain class of i.i.d. sources.

In this paper we make two uses of this procedure. First, in Section II, we refine their results, by a substantial weakening of the conditions on the density, thus calculating explicitly the rate distortion functions of larger classes of i.i.d. sources. In Theorem 1 the density of the source has finite support, 'Theorem 2 treats concave source densities, and in Theorem $1^{\prime}$ the support of the source density is the entire real line or a half line. We also indicate how Theorems 1 and $l^{\prime}$ may be combined with a known result ('Theorem $\Lambda$ ) in evaluating the rate distortion functions of certain i.i.d. sources whose densities do not satisfy the assumptions of Theorems 1 and 1'. Secondly, we develop a family of lower bounds for the rate distortion function of an arbitrary i.i.d. source (Theorem 3) and compare them with the Shannon lower bound in Section III.

* This research was supported by the Air Force Office of Scientific Research under Grant AFOSR-75-2796. 


\section{Rate Distortion Functions of I.I.D. Sources}

We first consider rate distortion functions of random variables with continuous densities which vanish outside a finite interval.

THEOREM 1. Let $X$ be a random variable with density $p(x)$ which vanishes outside the interval $[a, b],-\infty<a<b<\infty$. Assume the following:

(A) $p$ is continuous aith median $\mu$ and there is an at most finite set of points $a=d_{0}<d_{1}<\cdots<d_{m}<d_{m+1}=b(m \geqslant 0)$ such that on each $\left[d_{j}, d_{j+1}\right]$, $j=0,1, \ldots, m, p(x)$ is differentiable and its derivative $p^{\prime}(x)$ is absolutely continuous and satisfies $p_{-}^{\prime}\left(d_{j}\right) \geqslant p_{+}^{\prime}\left(d_{j}\right), j=1, \ldots, m$, where $p_{-}^{\prime}\left(d_{j}\right)$ and $p_{+}^{\prime}\left(d_{j}\right)$ are the left and right limits of $p^{\prime}$ at $d_{j}$ respectively. Also

$$
\int_{a}^{x} p(t) d t>0 \quad \text { for } \quad x>a ; \quad \int_{x}^{b} p(t) d t>0 \quad \text { for } x<b
$$

(B) The function

$$
K_{1}(x)=p(x) / \int_{x}^{b} p(t) d t \quad \text { for } \quad x \in[\mu, b)
$$

diverges to $\dashv-\infty$ as $x$ increases to $b$; and the function

$$
K_{2}(x)=p(x) / \int_{a}^{x} p(t) d t \quad \text { for } \quad x \in(a, \mu]
$$

diverges to $-; \infty$ as $x$ decreases to $a$.

Then for each $s \in(-\infty,-2 p(\mu))$, there exist unique $a_{s}>0$ and $b_{s}>0$ such that $a_{s} \downarrow \mu-a$ and $b_{s} \uparrow b--\mu$ as $s \downarrow-\infty$ and $a_{s}$ and $b_{s}$ are determined by

$$
\begin{aligned}
& \mu-a_{s}=\min \left\{y \in(a, \mu): K_{2}(y)=: s \mid\right\} \\
& \mu+b_{s}=\max \left\{y \in(\mu, b): K_{1}(y)=\{s \mid\} .\right.
\end{aligned}
$$

Suppose in addition that

(C) for each $s \in(-\infty,-2 p(\mu))$,

$$
p(x)-s^{-2} p^{\prime \prime}(x) \geqslant 0 \text { a.e. [Leb.] on }\left[\mu-a_{s}, \mu \div b_{s}\right] \text {. }
$$

Then the rate distortion function $R(D), 0<D<D_{\max }$, of $X$ is given parametrically in $s$ by

$$
\begin{aligned}
R\left(D_{s}\right)= & \ln \frac{|s|}{2}-\int_{\mu-a_{s}}^{L+b_{s}} p(x) \ln (e p(x)) d x-\ln \left(p\left(\mu-a_{s}\right)\right) \int_{a}^{\mu-a_{s}} p(x) d x \\
& -\ln \left(p\left(\mu-b_{s}\right)\right) \int_{\mu+b_{s}}^{b} p(x) d x
\end{aligned}
$$




$$
\begin{aligned}
D_{s}= & \frac{1}{|s|} \int_{\mu-a_{s}}^{\mu+b_{s}} p(x) d x+\int_{a}^{\mu-a_{s}}\left(\mu-a_{s}-x\right) p(x) d x \\
& +\int_{\mu+b_{s}}^{b}\left(x-\mu-b_{s}\right) p(x) d x
\end{aligned}
$$

where $-\infty<s<-2 p(\mu)$ and $D_{\max }=\int_{a}^{b}|x-\mu| p(x) d x$.

The proof of Theorem 1 is given in Appendix 1, and the following example is an application of 'Theorem 1 with non-monotonic $K_{1}(x)$.

EXample 1. I et $X$ be a random variable with density

$$
p(x)=(x \ln 100)^{-1}, \quad 0.01 \leqslant x \leqslant 1
$$

Then $p(x)$ is continuous and differentiable with $\mu=0.1$. Conditions (A) and (B) are clearly sarusfied. Note that $K_{1}(x)$ decreases for $\mu \leqslant x \leqslant e^{-1}$ and then increases to $+\infty$ as $x \uparrow 1$. Condition (C) is not satisfied for all $s$ but only for some $s$ in $(-\infty,-2 p(\mu))$. In this case, only a portion of $R(D)$ can be obtained (corresponding to those $D_{s}$ for which $s$ satisfies (C)). We have $p(x)-p^{\prime \prime}(x) / s^{2}=$ $p(x)\left(1-2 /\left(s^{2} x^{2}\right)\right) \geqslant 0$ if and only if $x \geqslant 2^{1 / 2} / ; \mid$. Thus only for large $|s|(\mathrm{C})$ will be satisfied. For $s:=-72.135$, we have $2^{1 / 2} /|s|==.0196$ and from (3) $\mu-a_{s}=0.02$. Thus $s \leqslant-72.135$ (C) is satisfied. This portion of $s$ corresponds to a region of small distortion $D$ (since $s$ is the slope of $R(D)$ ) and for this region $R(D)$ is given parametrically by (5) and (6).

We now show that certain continuous concave densities satisfy the assumptions of Theorem 1 and thus their rate distortion functions can be obtained explicitly.

Theorem 2. Let $X$ be a random variable with density $p(x)$ which vanishes outsice the interval $[a, b],-\infty<a<b<\infty$. Suppose $p(x)$ is a continuous concave function on $[a, b]$ and there is an at most finite set of points $a=d_{0}<$ $d_{1}<\cdots<d_{m}<d_{n+1}=b(m \geqslant 0)$ such that on each $\left[d_{j}, d_{j+1}\right], j=0, \ldots, m$, $p(x)$ is differentiable and its derivative is absolutely continuous. Then the rate distortion function of $X$ is given by (5) and (6).

Proof. Since $p(x)$ is concave, $p^{\prime \prime}(x) \leqslant 0$ and $p_{-}^{\prime}(x) \geqslant p_{+}^{\prime}(x)$. Also $\int_{a}^{x} p(t) d t>0$ for $x>a$. For suppose $\int_{a}^{x_{0}} p(t) d t=0$ for some $x_{0}>a$. Then $p(t)==0$ for each $t \in\left[a, x_{0}\right]$ by the continuity of $p$. 'Thus $p^{\prime}(t)=0$ for each $t \in\left[a, x_{0}\right]$. Since $p_{-}^{\prime}(t) \geqslant p_{+}^{\prime}(t)$, we have $p^{\prime}(t) \leqslant 0$ for cach $t \in[a, b]$. This implies $p(t)=0$ for $t \in[a, b]$ which is a contradiction. Similarly $\int_{x}^{b} p(t) d t>0$ for $x<b$. Thus the only assumption left to be verified in Theorem 1 is (B). 
If $p(b) \neq 0$, then it is clear from (1) that $K_{1}(x) \rightarrow+\infty$ as $x \hat{i} b$. Suppose now that $p(b)-0$. 'Then by l'Hospital's rule

$$
\lim _{x \uparrow b} K_{\mathbf{1}}(x)==\lim _{x \uparrow b}-p^{\prime}(x) ; p(x)
$$

We will show that $p^{\prime}(b)<0$ and thus $K_{1}(x) \rightarrow+\infty$ as $x \uparrow b$. By the concavity of $p(x), p^{\prime}(x)$ is a non-increasing function. Suppose $p^{\prime}(b) \geqslant 0$. Then $p^{\prime}(x) \geqslant 0$ for all $x \in[a, b]$ for which the derivative exists. Thus $p(x)$ is non-decreasing. Since $p(b)=0$, this implies that $p(x)=0$ for all $x \in[a, b]$, which contradicts the fact that $p$ is a density. Hence $p^{\prime}(b)<0$.

The proof of $K_{2}(x) \rightarrow \dashv-\infty$ as $x \downarrow a$ is of course similar.

It should be noted that it can also be shown that $K_{1}(x) \hat{\imath} \infty$ as $x \uparrow b$ and $K_{2}(x) \uparrow \infty$ as $x \downarrow a$.

COROLlaky 2.1. Let $X$ be a random variable with continuous density $p$ consisting of line segments and vanishing outside a finite interval. Then the rate distortion function of $X$ is given by (5) and (6) if and only if $p$ is concave.

Proof. It follows from 'Theorem 2 that if $p$ is concave then its rate distortion function is given by (5) and (6).

Now suppose that $p$ is not concave. 'Then there exist two adjacent line segments such that the left derivative at their common point is smaller than the right derivative. Hence for each $s, G_{s}(y)$ in the proof of Theorem 1 is not a probability distribution function and thus the parametric expressions (5) and (6) do not give the rate distortion function of $X$.

The following example is a direct application of Corollary 2.1. Calculation is straightforward and is omitted.

Example 2. Trapezoid density. If $0<c<a$ and

$$
p(x)= \begin{cases}(a+c)^{-1} & |x| \leqslant c \\ (a-\mid x):\left(a^{2}-c^{2}\right) & c \leqslant|x| \leqslant a\end{cases}
$$

then for $0<D \leqslant(a-c)(a+2 c) i 3(a \div c)$

$$
\begin{aligned}
R(D)= & \left.2 \cos ^{2}\left[\frac{4 \pi}{3}+\frac{1}{3} \cos ^{-1}(-3 D)\left(a^{2}-c^{2}\right)^{1 / 2}\right)\right]-(a \cdot 3 c) 2(a \cdot c) \\
& -\ln \left(2((a-c):(a+c))^{1 / 2} \cos \left[\frac{4 \pi}{3} \cdot i \frac{1}{3} \cos ^{-1}\left(--3 D\left(a^{2}-c^{2}\right)^{1 / 2}\right)\right]\right)
\end{aligned}
$$




$$
\begin{gathered}
\text { and for }(a-c)(a+2 c) / 3(a+c) \leqslant D \leqslant D_{\max }=:=\left(a^{3}-c^{3}\right) / 3\left(a^{2}-c^{2}\right) \\
R(D)=-f(D)-\ln (1-f(D))
\end{gathered}
$$

where $f(D)=\left[1-4 D /(a-c)-(a-c)^{2} / 3(a-c-c)^{2}\right]^{1 / 2}$.

Theorem 1 is also valid when the support of $p(x)$ is not finite. The result is stated in the following:

'THeOrem $1^{\prime}$. Let $X$ be a random variable with density $p(x)$. If $p(x)$ satisfies all assumptions in Theorem 1 with $\cdots \infty \leqslant a<b \leqslant+\infty$, then the rate distortion function of $X$ is given by (5) and (6) with $-\infty \leqslant a<b \leqslant-\infty$.

Theorem $1^{\prime}$ is an improvement of Theorem 3 in ['T'an and Yao, (1975)]. Here we no longer require the monotonicity of $K_{i}(x), i=1,2$, and we allow $p^{\prime}(x)$ to have a finite number of discontinuities instead of a single discontinuity at $\mu$.

The following (known) result can be used along with Theorems 1 and $1^{\prime}$ in evaluating the rate distortion functions of certain random variables.

'THEOREM A. If the random variable $X_{i}$, has distribution function $F_{i}$, and distortion rate function $D_{i}(R), i:=1,2$, then for all $R>0$,

$$
\left|D_{1}(R)-D_{2}(R)\right| \leqslant \int_{-\infty}^{\infty}\left|F_{1}(t)-F_{2}(t)\right| d t .
$$

Thus if $F_{n}-F \rightarrow 0$ in $L_{1}$, or if $F_{n} \rightarrow F$ weakly and $F_{n}, F$ have finite means, then $D_{n}(R) \rightarrow D(R)$ uniformly.

Proof. Corollary 1 of [Gray, Neuhoff and Shields (1975)] applied to i.i.d. sources with distributions $F_{1}$ and $F_{2}$ gives

$$
\left|D_{1}(R)-D_{2}(R)\right| \leqslant \bar{\rho}\left(F_{1}, F_{2}\right) .
$$

But by [Vallender (1973)] $\bar{\rho}\left(F_{1}, F_{2}\right)=\int_{-\infty}^{\infty}, F_{1}(t)-F_{2}(t) \mid d t$ and thus (10) follows. Now if $F_{n}$ converges to $F$ weakly and all distributions involved have finite means, then by Theorem 2 of [Dobrushin (1970)], we have $\bar{\rho}\left(F_{n}, F\right) \rightarrow 0$ and hence $D_{n}(R) \rightarrow D(R)$ uniformly.

The following well-known property is useful in connection with Theorcm A: If a sequence of probability density functions $p_{n}$ converges to a probability density function $p$ almost everywhere, then the correponding sequence of distributions $F_{n}$ converges to the distribution $F$ of $p$ weakly. For example, suppose $p(x)$ is a truncated double exponential density defined on $[-c, c]$, $c>0$, i.e.

$$
p(x)=-\alpha \exp \left(-\alpha_{1}^{\prime} x \mid\right) / 2(1-\exp (-\alpha c)) \quad|x| \leqslant c, \quad \alpha>0 .
$$


Then a direct application of 'Iheorem 1 yields

$$
\begin{aligned}
& \left.R(I)_{s}\right) \ldots \ln [\mid s !(1-\exp (-\alpha c)) ; \alpha] \\
& -\alpha \exp (\cdots \alpha c)\left[c \quad \vdots \alpha^{-1} \ln (1 \cdots \alpha / s:)\right] /[1-\exp (-\alpha c)] \\
& D_{s}=\left[\because s ! 1 \ldots \cdot \alpha^{-1} \exp (-\alpha c) \ln (1-\alpha /|s|)\right]_{i}[1-\ldots \exp (-\alpha c)]
\end{aligned}
$$

where: $s: \epsilon\left[\alpha_{i}^{i}(1-\exp (-\alpha c)), \infty\right)$ and

$$
D_{\max }=\left[\alpha^{1}-\left(c+\alpha^{1}\right) \exp (-\alpha c)\right][1-\exp (-\alpha c)]
$$

Now by letting $c>\infty$ in (11) (14), we find the rate distortion function for the double exponential density on the entire real line (since all distributions involved have finite means), i.e. $R(D)=-\ln \alpha D, 0<D \leqslant \alpha^{-1}=-D_{\max }$. Of course, this has been calculated by using the Shannon lower bound method [Berger (1971), p. 95]. Note that the double exponential density does not sat isfy assumption $(B)$ of 'Theorem 1', while the truncated double exponential densities satisfy all the assumptions of Theorem 1 . This demonstrates how Theorems 1 and $A$ can be used in evaluating the rate distortion function of certain random variables and following are some further examples.

Example, 3. (a) Triangular density: Letting $c \downarrow 0$ in (7) we see that the trapezoid density converges to the triangular density $(a-x) ; a^{2},{ }^{\prime} x \mid \leqslant a$. Its rate distortion function is then found by letting $c{ }_{y} 0$ in $(8)$ : for $0<D \leqslant$ a/3 $=D_{\max }$,

$$
\begin{aligned}
R(D) \ldots 2 \cos ^{2}\left[\frac{4 \pi}{3}: \frac{1}{3} \cos ^{-1}\left(-\frac{3 D}{a}\right)\right] \\
\quad-\frac{1}{2}-\ln \left(2 \cos \left[\frac{4 \pi}{3}-\frac{1}{3} \cos ^{-1}\left(-\frac{3 D}{a}\right)\right]\right) .
\end{aligned}
$$

(b) Lniform density: Letting $c \uparrow a$ in (7) we see that the trapezoid density converges to the uniform density $l /(2 a), \mid x \leqslant a$, whose rate distortion function is thus found by letting $c \uparrow a$ in (9): for $0<D \leqslant a: 2:=D_{\max }$,

$$
R(D) \quad-\left(1-\frac{2 D}{a}\right)^{1 / 2} \cdots \ln \left(1 \cdots\left(1-2 D-\frac{2 D}{a}\right)^{1 / 2}\right)
$$

Note that the triangular and uniform densities satisfy all assumptions of 'Theorem 1 and thus (15) and (16) could be obtaincd directly from (5) and (6). (16) was first given by 'Tan and Yao (1974).

If the method used in Theorem 1 is applied to a discontinuous probability density function, a $\lambda_{s}(x) \geqslant 0$ may be found satisfying $c_{s}(y) \leqslant 1$ whereas a distribution function $G_{\mathrm{s}}(y)$ satisfying $(\Lambda .3)$ of Appendix 1 may not exist. In this 
case, using the above mentioned $\lambda_{s}$, a lower bound $R_{L}(D)$ for the rate distortion function $R(D)$ of the discontinuous density can be obtained. The following example illustrates this point.

EXAMPIE 4. Let

$$
p(x)== \begin{cases}1 / 4 & -1 \leqslant x<0 \\ 3 / 8 & 0 \leqslant x \leqslant 2\end{cases}
$$

Then for $0<D \leqslant 9 / 16$, we have

$$
R_{L}(D)=-\frac{1}{2}(4-5 D)^{1 / 2}-\ln \left(2-(4-5 D)^{1 / 2}\right)-\frac{1}{4} \ln (54 / 625)
$$

and for $13 / 24 \leqslant D \leqslant 17 / 24=D_{\max }, R(D)$ itself can be found and is given by

$$
R(D)=-(1-(24 D-1) / 16)^{1 / 2}-\ln \left(1-(1-(24 D-1) / 16)^{1 / 2}\right) .
$$

Theorem A may yet be used in another way to find bounds for distortion rate functions of discontinuous densities.

Example 5. Let $p(x)$ be the density of Example 4 and, for $0<\epsilon<1$, consider the continuous approximating density

$$
p_{\epsilon}(x)=\left\{\begin{array}{lr}
14 & -1 \leqslant x \leqslant-\epsilon \\
x^{2} / 16 \epsilon^{2} \rightarrow x: 8 \epsilon+5 ; 16 & -\epsilon \leqslant x \leqslant 0 \\
-x^{2} / 16 \epsilon^{2}-x: 8 \epsilon+5: 16 & 0 \leqslant x \leqslant \epsilon \\
3 ; 8 & \epsilon \leqslant x \leqslant 2
\end{array}\right.
$$

Note that $p_{\epsilon}(x)$ converges to $p(x)$ almost everywhere as $\epsilon \rightarrow 0$. In this case the $R_{\epsilon}(D)$ of $p_{\epsilon}$ can be cvaluated by Theorem 1 and for $\epsilon=: 0.59367$, we have

$$
D_{\epsilon}(R)-0.00367 \leqslant D_{L}(R) \leqslant D(R) \leqslant D_{\epsilon}(R)+0.00367
$$

where $I_{L}(R)$ is the inverse function of $R_{L}(D)$ in Example 4. (For the evaluation of $D_{\epsilon}(R)$ see Leung (1976).)

\section{Bocnds to Rate Distortion Functions}

In this section (further) bounds are derived for rate distortion functions of random variables whose densities do not satisfy all the assumptions in the theorems of Section II. Examples are then given comparing these bounds with the Shannon lower bound. 
I'HFOREM 3. Iet $X$ be a random variable with density $p(x)$ satisfying the assumptions in Theorem 1 . Let $X_{1}$ be another random zariable whose density $p_{1}(x)$ vanishes outside the interval $[a, b]$ and has at most a finite number of simple discontinuities. Then a lower bound for the rate distortion function of $X_{1}$ is given parametrically in s by

$$
\begin{aligned}
& R_{L}\left(D_{s}\right):-H_{\rho}\left(p_{1}\right)+\ln \frac{|s|}{2}-\ln \left(p\left(\mu-a_{s}\right)\right) \int_{a}^{\mu-a_{s}} p_{1}(x) d x \\
&-\int_{\mu-a_{s}}^{\mu-b_{s}} p_{1}(x) \ln (e p(x)) d x-\ln \left(p\left(\mu-; b_{s}\right)\right) \int_{\Delta \leq b_{s}}^{b} p_{1}(x) d x \\
& D_{s}=\int_{a}^{\mu-a_{s}}\left(\mu-a_{s}-x\right) p_{1}(x) d x+\frac{1}{|s|} \int_{\mu-a_{s}}^{\mu-b_{s}} p_{1}(x) d x \\
& \quad-\int_{\mu-b_{s}}^{b}\left(x \cdots \mu-b_{s}\right) p_{1}(x) d x
\end{aligned}
$$

where $H_{n}\left(p_{1}\right)=\int_{a}^{b} p_{1}(x) \ln \left(p_{1}(x) / p(x)\right) d x$ is the generalized entropy of $p_{1}$ with respect to $p$ [Pinkser (1964), p. 18]; $\mu$ is the median of $p$ and $a_{s}$ and $b_{s}$ are related to $s$ by (3) and (4).

The proof of 'Iheorem 3 is given in Appendix 2. It should be noted that $H_{p}\left(p_{1}\right) \geqslant 0$, with equality if and only if $p(x)=p_{1}(x)$ a.e. [Pinsker (1964), p. 19], and that $R_{L}$ is useful only when $H_{p}\left(p_{1}\right)<\infty$. Clearly Theorem 3 is also valid when the support of the densities is not finite. Necessary and sufficient conditions for the lower bound $R_{L}(D)$ of Theorem 3 to equal $R(D)$ are given in the following theorem whose proof is given in Appendix 3 .

Theorem 4. For each fixed $s \leqslant 0, R_{L}\left(D_{s}\right)$ given in Theorem 3 is equal to $R\left(D_{s}\right)$ if and only if there exists a probability distribution function $Q_{s}$ whose total probability is concentrated on (a subset of) $[a, b]$ and is such that

$$
p_{1}(x)- \begin{cases}\frac{|s| e^{-s\left(\mu-a_{s}-x\right)} p(x)}{2 p\left(\mu-a_{s}\right)} \int_{a}^{b} e^{s ; x-y} d Q_{s}(y), & x \in\left[a, \mu-a_{s}\right), \\ \frac{|s|}{2} \int_{a}^{b} e^{s i x-y \mid} d Q_{s}(y), & x \in\left[\mu-a_{s}, \mu-\therefore-b_{s}\right], \\ \frac{|s| e^{s\left(\mu+b_{s}-x\right)} p(x)}{2 p\left(\mu-b_{s}\right)} \int_{a}^{b} e^{s|x-y|} d Q_{s}(y), & x \in\left(\mu-\because-b_{s}, b\right],\end{cases}
$$

where $a_{s}$ and $b_{s}$ are given by (3) and (4), and

$$
\int_{a}^{b} \lambda_{s}(x) p(x) \exp (s|x-y|) d x=1 \quad \text { a.e. } \quad\left[d Q_{s}\right]
$$


It would be of interest to compare the lower bound of Theorem 3 with the well known Shannon lower bound for densities which vanish outside the interval $[a, b],-\infty<a<b<\infty$.

'Theoria B. Let $X$ be a random variable with density $p(x)$ which vanishes outside $[a, b],-\infty<a<b<\infty$. Then the Shannon lower bound to $R(D)$ of $X$ is given parametrically in $s<0$ by

$$
\begin{aligned}
R_{S L}\left(D_{s}\right) & =h(p)-|s|(b-a) / 2 k(s)+-\ln |s| / 2 e k(s) \\
D_{s} & =|s|^{\cdots 1}+(b-a) / 2 k(s)
\end{aligned}
$$

where $h(p)=-\int_{a}^{b} p(x) \ln p(x) d x$ and $k(s)=1-\exp (|s|(b-a)$;). Moreover, $R_{S L}(D)<R(D)$ for all $D$ with $R(D)>0$ unless

$$
p(x)=\mid s ; \exp (s|x-(a+b) / 2| 2) / 2 k(s), \quad x \in[a, b],
$$

in which case $R_{S I}\left(D_{0}\right)==R\left(D_{0}\right)$ at the point $D_{0}$ with slope s.

EXAMPLE 6 . Let $p(x)$ be the uniform density on $[a, b]$ which satisfies all assumptions in Theorem 1, and let $p_{1}(x)$ be a piecewise continuous density on $[a, b]$. 'Then by applying Theorem 3 , a lower bound for $R(D)$ of $p_{1}$ can be found. Evaluation of (17) and (18) shows that for $s<-(b-a)^{-1}$

$$
\begin{aligned}
R_{L}\left(D_{s}\right) & =h\left(p_{1}\right)+|\ln | s \mid: 2 e-\int_{0}^{\left.s\right|^{-1}}\left[p_{1}(a \nmid t)+p_{1}(b-t)\right] d t \\
D_{s}:= & |s|^{-1}-\int_{0}^{s s^{-1}} t\left[p_{1}(a-t) \vdash p_{1}(b-t)\right] d t .
\end{aligned}
$$

In (25), if for a given $D,|s|$ is not single-valued, and if a branch of $|s|$ can be chosen such that (A.21) in Appendix 2 is satisfied, then for this branch of $|s|$, $R_{L}(I)$ is the best possible lower bound achicved by the method of Theorem 3 . Note that for this example condition (A.21) is equivalent to

$$
p_{1}\left(a:-|s|^{-1}\right)+p_{1}\left(b-! s i^{-1}\right)<i s \mid .
$$

The lower bound of Theorem 3 is of course useful when Theorem 1 is not applicable to $p_{1}$. As an illustration we now calculate the lower bound of Theorem 3 when $p_{1}$ is the truncated double exponential density given by (11). In this case the rate distortion function of $p_{1}$ is given by (12)-(14) and therefore we can see how tight is the lower bound determined by (24) and (25). Calculations show the following: 


$$
\begin{aligned}
h(p) & =1-\frac{\alpha}{2}+\ln 2 u(\alpha)-\frac{1}{2} u(\alpha)^{-1} \\
R_{L}\left(D_{s}\right) & =-\frac{\alpha}{2}+\ln |s| u(\alpha)+[2 \exp (\alpha|s|)-\alpha-2] / 2 \alpha u(\alpha) \quad|s|>2 \\
D_{s} & -\mid s_{:}^{-1}-[\alpha u(\alpha)]^{-1}\left[\left(|s|^{-1}-\alpha^{-1}\right) \operatorname{cxp}(\alpha ; i \mid)+\alpha^{-1}\right] \\
D E O & =\left[\frac{1}{\alpha}-\left(1 \div \alpha^{-1}\right) \exp (\alpha ; 2)\right] /[1-\exp (-\alpha ; 2)]
\end{aligned}
$$

where $u(\alpha)=-=[\exp (\alpha / 2)-1] / \alpha$. For the Shannon lower bound we have

$$
\begin{aligned}
\operatorname{Re}_{L}\left(D_{s}\right) & =(|s|-\alpha) 2-\frac{1}{2} u(\alpha)^{-1}+\ln 2 u(\alpha)+\ln v(s)+v(s) & \\
D_{s} & =|s|^{-1}(1-v(s)) . & |s|>0
\end{aligned}
$$

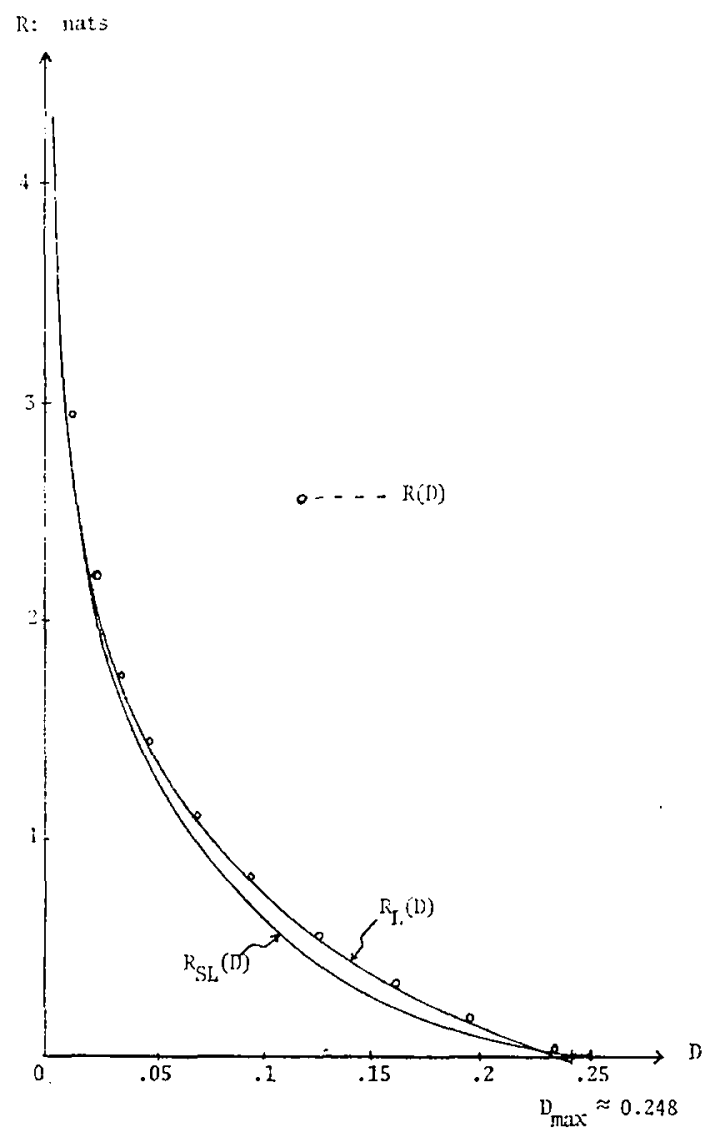

Fig. 1. $a=0.1$. 


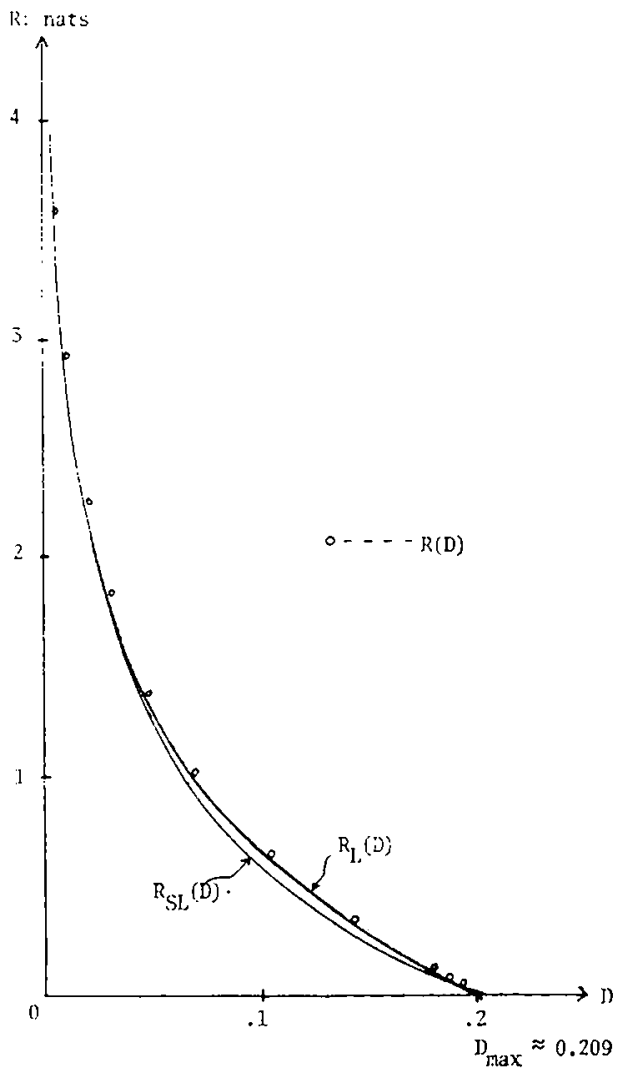

Fig. 2. $\alpha=2$.

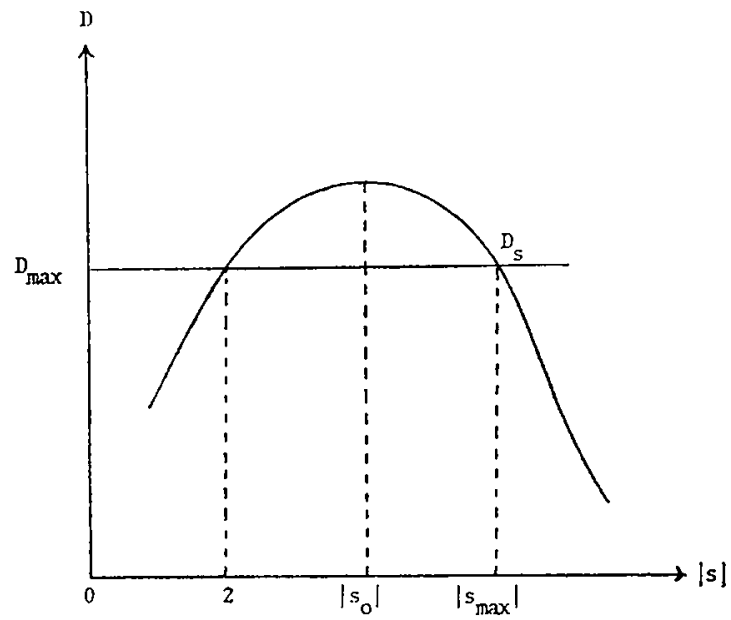

FIG. 3 
where $v(s) \ldots s / 2(\exp (\mid s ; 2)-1)$. Curves are plotted for $x-0.1,2$ in Figures 1 and 2. In general, $R_{L}$ is a better lower bound than $R_{S L}$, except is a small neighborhood of $D_{\max }$ where $R_{S L}$ is better. As $x \rightarrow 0$, the difference between $R_{L}$ and $R_{S L}$, becomes larger and as $\alpha \rightarrow \infty$, the difference becomes smaller. It can also be seen that $R_{L}$ is a very good approximation to $R$. If, for $\alpha>0$ fixed, we plot $D$ as a function of $|s|$ as given by (27), we obtain a curve as shown in Figure 3. Note that at $|s|=2, D_{s}=D_{\max }$. Also $D_{s}$ achieves its maximum at some point $\left|s_{0}\right|>2$, and $D_{s}$ is a decreasing function for all $\mid s ; \geqslant\left\{s_{0} \mid\right.$. It follows (as it is easily checked analytically) that for all $|s| \geqslant$ $s_{\max } \mid$, condition (26) is satisfied and thus the branch of $; s^{1}: ! s|>| s_{\max }$ ! gives the tightest possible lower bound $R_{L}\left(D_{s}\right)$.

Another lower bound for the rate distortion function of the truncated double exponential density can be obtained by using the truncated Gaussian density and numerical calculations show that this lower bound is even slightly better than the one obtained using the uniform distribution.

\section{APPENDIXFS}

\section{Proof of Theorem 1}

The well-known analytical expression of $R(D), D>0$, is given by [Berger (1971)]

$$
R(D)=\sup _{s \leqslant 0, \lambda_{s} \in A_{s}}\left[s D+\int_{-\infty}^{\infty} p(x) \ln \lambda_{s}(x) d x\right]
$$

where $\lambda_{s}$ is the set of all non-negative functions $\lambda_{s}$ satisfying

$$
C_{s}(y)=\int_{-\infty}^{\infty} \lambda_{s}(x) p(x) \exp \left(s_{i} x-y_{i}\right) d x \leqslant 1 \quad \text { for all } y .
$$

Moreover, for fixed $s \leqslant 0$, the supremum in (A.l) is attained by a $\lambda_{s}$ if there is a distribution $G_{s}$ with support $V_{s} \subset[a, b]$ such that

$$
[\lambda(x)]^{-1}=\int_{V_{s}} \exp (s|x-y|) d G_{s}(y)
$$

and $C_{s}(y)=1$ for all $y \in V_{s}$. Then $R(D)$ is expressed parametrically in $s$ by

$$
\begin{aligned}
R\left(D_{s}\right) & =s D_{s}+\int_{-\infty}^{\infty} p(x) \ln \lambda_{s}(x) d x \\
D_{s} & =\int_{-\infty}^{\infty} \int_{-\infty}^{\infty} \lambda_{s}(x) p(x) ; x-y \mid \exp (s ; x-y \mid) d x d G_{s}(y) .
\end{aligned}
$$


Following the procedure of ' $T$ an and Yao (1975), we let $V_{s}=\left[\mu-a_{s}, \mu \cdot f \cdot b_{s}\right]$ be a subinterval of $[a, b]$, and we specify $a_{s}$ and $b_{s}$ (and $G_{s}$ ) so that (A.3) is satisfied and $C_{s}(y)=1$ for $y \in V_{s}$ and $C_{s}(y) \leqslant 1$ for $y \notin V_{s}$.

As was proved by 'Tan and Yao, (1975), $C_{s}(y)=1$ for $y \in V_{s}$ and (A.3) are equivalent to (A.3) and (A.6) to (A.8):

$$
\begin{aligned}
\int_{V_{s}} \exp (s|x-t|) d G_{s}(t) & =(2 i|s|) p(x), \quad x \in V_{s}, \\
\int_{a}^{\mu-a_{s}} p(t) d t & =\frac{1}{|s|} p\left(\mu-a_{s}\right), \\
\int_{\mu+b_{s}}^{b} p(t) d t & =\frac{1}{|s|} p\left(\mu+b_{s}\right) .
\end{aligned}
$$

Now (A.7) is equivalent to $K_{2}\left(\mu-a_{s}\right)=; s \mid$. Note that conditions (A) and (B) imply that $K_{2}(x)$ is continuous on $(a, \mu]$, differentiable on $(a, \mu]$ except at those $d_{j}$ 's which belong to $(a, \mu]$ at which left and right derivatives exist, and satisfies $K_{2}(\mu)==2 p(\mu)$ and $\lim _{x \downarrow a} K_{2}(x)=+-\infty$. It follows that given any $s \in(-\infty, 2 p(\mu))$ the equation $K_{2}\left(\mu-a_{s}\right)==s^{\prime}$, has at least one solution. For reasons which will become clear later on in this proof we will choose the smallest solution:

$$
\mu-a_{s}=\min \left\{y \in(a, \mu): K_{2}(y)=|s|\right\}
$$

which is clearly such that $\mu-a_{s} \downarrow a$ as $s !-\infty$ and has the following propertics (to be used later on):

$$
K_{2, \div}^{\prime}\left(\mu-a_{s}\right) \leqslant 0, \quad \text { and } \quad K_{2}(y)>|s| \quad \text { for all } y \in\left(a, \mu-a_{s}\right) \text {. }
$$

Similarly, by the properties of $K_{1}(x), b_{s}$ is uniquely determined by

$$
\mu+b_{s} \therefore \max \left\{y \in(\mu, b): K_{1}(y)=\mid s_{i}\right\}
$$

and has the following properties: $\mu-! \cdot b_{s} \uparrow b$ as $s \downarrow-\infty$,

$$
K_{1,-}^{\prime}\left(\mu+b_{s}\right) \geqslant 0, \quad \text { and } \quad K_{1}(y)>|s| \quad \text { for all } y \in\left(\mu+b_{s}, b\right) \text {. }
$$

We next show that for each $s \in(-\infty,-2 p(\mu))$, the distribution function $G_{s}(x)$ which has absolutely continuous part with density $p(x)-s^{-2} p^{\prime \prime}(x)$ on $V_{s}$ and zero elsewhere, discrete part with atoms at the points $\mu-\alpha_{s}, \mu+b_{s}$ and the $d_{j}$ 's which arc in $\left[\mu-a_{s}, \mu-b_{s}\right]$ and masses to be determined, and zero continuous singular part, is a solution of (A.6). For notational convenience we 
will work with the "density" $g_{s}$ of the above described distribution function $G_{s}$ which is thus of the form

$$
\begin{aligned}
g_{s}(t) \quad & p(t)-s^{-2} p^{\prime \prime}(t) \div C_{1, s} \delta\left(t-\mu ; \cdot a_{s}\right) \\
& +C_{2, s} \delta\left(t-\mu-b_{s}\right) \div \sum_{j=l}^{l+n-1} D_{j, s} \delta\left(t-d_{j}\right)
\end{aligned}
$$

for $t \in V_{s}$ and zero elsewhere, where $d_{l-1}<\mu-a_{s} \leqslant d_{l}<\cdots<d_{l+n-1} \leqslant$ $\mu-b_{s}<d_{l+n}$ and $\delta$ is the Dirac Delta function. A straightforward calculation gives the values of the masses $C_{1 . s}, C_{2, s}$ and $D_{j, s}$ so that (A.6) is satisfied:

$$
\begin{aligned}
& C_{1, s}=|s|^{{ }^{2}}\left[|s| p\left(\mu-a_{s}\right)-p_{+}^{\prime}\left(\mu-a_{s}\right)\right], \\
& C_{2, s}=|s|^{-2}\left[|s| p\left(\mu \div b_{s}\right)-p_{-}^{\prime}\left(\mu+b_{s}\right)\right], \\
& D_{j, s}=|s|^{-2}\left[p_{-}^{\prime}\left(d_{j}\right)-p_{+}^{\prime}\left(d_{j}\right)\right], \quad l \leqslant j \leqslant l \div n-1 .
\end{aligned}
$$

Having determined $g_{s}$ so as to satisfy (A.6) it remains to be shown that $g_{s}$ is a probability "density" function, i.e. that $G_{s}$ is a probability distribution function. Since $p(t)-s^{-2} p^{\prime \prime}(t) \geqslant 0$ a.e. [Leb] on $V_{s}$ by assumption (C) and $p_{-}^{\prime}\left(d_{j}\right)-$ $p_{i}^{\prime}\left(d_{j}\right) \geqslant 0$ by assumption (A), it is clear from the expressions (A.11) and (A.12) that $G_{s}$ is a distribution function if and only if

$$
\begin{aligned}
& |s| p\left(\mu-a_{s}\right)-p_{+}^{\prime}\left(\mu-a_{s}\right) \geqslant 0 \\
& |s| p\left(\mu-b_{s}\right)+p_{-}^{\prime}\left(\mu+\dot{b}_{s}\right) \geqslant 0
\end{aligned}
$$

and

$$
\int_{V_{s}} g_{s}(t) d t=\int_{V_{s}} d G_{s}(t)=1
$$

To show (A.13), we proceed as follows. Since $K_{2,+}\left(\mu-a_{s}\right) \leqslant 0$ we have from (2)

$$
p^{\prime}\left(\mu-a_{s}\right) \int_{a}^{\mu-a_{s}} p(t) d t-p^{2}\left(\mu-a_{s}\right) \leqslant 0
$$

and using (A.7) we obtain

$$
\left[\int_{a}^{\mu-a_{s}} p(t) d t\right]\left[p_{+}^{\prime}\left(\mu-a_{s}\right)-|s| p\left(\mu-a_{s}\right)\right] \leqslant 0 .
$$

Now since $a<\mu-a_{s}$ we have $\int_{a}^{\mu-a_{s}} p(t) d t>0$ by condition (A) and thus (A.13) follows. (A.14) can be proved similarly, and (A.15) can be verified easily. 
Next we show that $C_{s}(y) \leqslant 1$ for $y \& V_{s}$. Substituting (A.11) and (A.12) into (A.3) we find

$$
\lambda_{s}(x)= \begin{cases}{\left[! s \mid / 2 p\left(\mu-a_{s}\right)\right] \exp \left(-s\left(\mu-a_{s}\right):-s x\right)} & x \in\left[a, \mu-a_{s}\right] \\ |s| i 2 p(x) & x \in V_{s} \\ {\left[|s| / 2 p\left(\mu \div b_{s}\right)\right] \exp \left(s\left(\mu ; b_{s}\right)-s x\right)} & x \in\left[\mu+b_{s}, b\right]\end{cases}
$$

Now suppose that $a \leqslant y<\mu-a_{s}$. Then

$$
C_{s}(y)=\int_{a}^{y} \lambda_{s}(x) p(x) \exp (s(y-x)) d x-!-\int_{y}^{b} \lambda_{s}(x) p(x) \exp (s(x-y)) d x .
$$

Differentiating $C_{s}(y)$ with respect to $y$, we have

$$
\begin{aligned}
C_{s}^{\prime}(y) & =-|s| \int_{a}^{y} \lambda_{s}(x) p(x) \exp (s(y-x)) d x+-|s| \int_{y}^{b} \lambda_{s}(x) p(x) \exp (s(x-y)) d x \\
& =|s|\left(C_{s}(y)-h_{s}(y)\right)
\end{aligned}
$$

where

$$
h_{s}(y)=2 \exp (s y) \int_{a}^{y} \lambda_{s}(x) p(x) \exp (-s x) d x
$$

Substituting (A.16) into (A.18) we have

$$
\begin{aligned}
h_{s}(y) & =|s| \exp \left(-s\left(\mu-a_{s}\right)+s y\right) \int_{a}^{y} p(x) d x / p\left(\mu-a_{s}\right) \\
& =\left[\exp (s y) \int_{a}^{y} p(x) d x\right] /\left[\exp \left(s\left(\mu-a_{s}\right)\right) \int_{a}^{\mu-a_{s}} p(x) d x\right]
\end{aligned}
$$

We now show that

$$
f(y)=\exp (s y) \int_{a}^{y} p(t) d t, \quad y \in\left(a, \mu-a_{s}\right]
$$

is increasing. Indeed we have

$$
\left.f^{\prime}(y)=\exp (s y)(p(y)-\mid s\} \int_{a}^{y} p(t) d t\right) .
$$

Now (A.9) and (2) imply, as was remarked, that

$$
K_{2}(y) \geqslant K_{2}\left(\mu-a_{s}\right)=\mid s ! \quad \text { for } \quad a<y \leqslant \mu-a_{s} .
$$

It then follows from (2) that $f^{\prime}(y) \geqslant 0, a<y \leqslant \mu-a_{s}$, and thus $f$ is increasing 
on $\left(a, \mu \cdots a_{\mathrm{s}}\right]$. Hence $h_{\mathrm{s}}(y) \leqslant 1$ for $y \in\left(a, \mu-a_{s}\right]$ and since $h_{\mathrm{s}}(a):=0$, it follows that $h_{s}(y) \leqslant 1$ for $y\left[a, \mu-a_{s}\right]$. Now, as in Appendix $A$ of Tan and Yao (1975), it is shown that $C_{s}(y) \leqslant 1$ for all $y \notin V_{s}$.

Thus $R(D)$ is given parametrically by (A.4) and (A.5) which have the final expressions given in (5) and (6) as was shown in Appendix C of Tan (1973).

\section{Proof of Theorem 3}

Since $p$ satisfies the assumptions of 'Theorem $1, \lambda_{s}(x)$ given by (A.16) satisfies

$$
C_{s}(y)=\int_{a}^{b} \lambda_{s}(x) p(x) \exp (s|x-y|) d x \leqslant 1, \quad \text { for all } y \in[a, b] .
$$

Now define $\lambda_{s}^{(1)}(x)$ by

$$
\lambda_{s}^{(1)}(x) p_{1}(x)=\lambda_{s}(x) p(x) .
$$

Then $\lambda_{s}^{(1)}(x)$ also satisfies

$$
C_{s}(y)=\int_{a}^{b} \lambda_{s}^{(1)}(x) p_{1}(x) \exp (s|x-y|) d x \leqslant 1, \quad \text { for all } y \in[a, b] .
$$

By (A.1), $\lambda_{s}^{(1)}(x)$ yields a lower bound to the rate distortion function of $X_{1}$, i.e.

$$
\sup _{s \leqslant 0}\left(s D:-\int_{a}^{b} p_{1}(x) \ln \lambda_{s}^{(1)}(x) d x\right) .
$$

Let $R_{L}(D, s) \cdots s D+\int_{a}^{b} p_{1}(x) \ln \lambda_{s}^{(1)}(x) d x$ and let $d_{j}, j=1, \ldots, n, a=d_{0}<$ $d_{1}<\cdots<d_{n}<d_{n+1}-b$ be the points where $p_{1}$ has simple discontinuities. Then from (A.19), (A.16) and the existence of $\partial \lambda_{s}(x) / \partial s, p_{1}(x) \ln \lambda_{s}^{(1)}(x)$ is continuous both in $s$ and $x$ and its partial derivative with respect to $s$ exists for each $x \in[a, b]$ and $s \leqslant 0$ and is bounded by a constant. Thus

$$
\therefore R_{L}(D, s)=D+\left\{\int_{a}^{d_{1}}+\frac{n}{\partial s}+\sum_{j=1}^{n-1} \int_{d_{j}}^{d_{j+1}}+\int_{d_{n}}^{b}\right\} \frac{p_{1}(x)}{\lambda_{s}^{(1)}(x)} \frac{\partial \lambda_{s}^{(1)}(x)}{\partial s} d x
$$

Setting $\left.\partial R_{I}(I), s\right) / \partial s-0$ and substituting (A.19) in the above expression, we have

$$
D_{s}=-\int_{a}^{b} \frac{p_{1}(x)}{\lambda_{s}(x)} \frac{\partial \lambda_{s}(x)}{\partial s} d x
$$

'Thus for each fixed $D$, if

$$
\partial^{2} R_{L}(D, s) / \partial s^{2} \leqslant 0 \quad \text { for all } \quad s \leqslant 0
$$


then $R_{L}(D, s)$ as a function of $s$ is concave and its supremum is achicved by the point $s_{D}$ satisfying (A.20), i.e.

$$
R_{L}\left(D, s_{D}\right)=\sup _{s \leqslant 0} R_{L}(D, s)
$$

Whether or not (A.21) is satisfied, $R_{L}\left(D_{s}\right):=R_{L}\left(D_{s}, s\right)$ along with (A.20) provide the parametric expressions (in $s$ ) of a lower bound of the rate distortion function of $X_{1}$. Substituting the expression of $\partial \lambda_{s}(x) / \partial s$ [see Tan and Yao (1975)] into (A.20), we obtain (18), and substituting (18) and (A.16) into (A.4), we obtain (17).

\section{Proof of Theorem 4}

Suppose the assumptions are satisfied for a given $s \leqslant 0$. Substituting (A.16) into (19) and using (A.19), we have

$$
\begin{aligned}
p_{1}(x) & =\lambda_{s}(x) p(x) \int_{a}^{b} \exp (s|x-y|) d Q_{s}(y) \\
& =\lambda_{s}^{(1)}(x) p_{1}(x) \int_{a}^{b} \exp (s|x-y|) d Q_{s}(y) .
\end{aligned}
$$

If $p_{1}(x): \neq 0$, then we have

$$
\left[\lambda_{s}^{(1)}(x)\right]^{-1}=\int_{a}^{b} \exp (s|x-y|) d Q_{s}(y)
$$

If $p_{1}\left(x_{0}\right)=0$ for some $x_{0} \in[a, b]$, then from (19), $p_{1}\left(x_{0}\right)=p\left(x_{0}\right)==0$ for some $x_{0} \in\left[a, \mu-a_{s}\right) \cup\left(\mu \div b_{s}, b\right]$. In this case, we can define $\lambda_{s}^{(1)}(x)$ by (A.22). Therefore $R_{L}\left(D_{s}\right)=R\left(D_{s}\right)$. Conversely, for a given $s \leqslant 0$, suppose $R_{L}\left(D_{s}\right)=$ $R\left(D_{s}\right)$. Then $\lambda_{s}^{(1)}(x)$ achieves the supremum in (A.1) and hence it satisfies (A.3), i.e. (A.22), and is such that $C_{s}(y)=1$ a.e. $\left[d Q_{s}(y)\right]$ for some probability distribution $Q_{s}$. Substituting (A.19) and (A.16) into (A.22), we obtain (19).

\section{ACKNOWLEDGMENT}

A reviewer pointed out that related work can be found in Yao, K. and Tan, H. H., Absolute error rate-distortion functions for sources with constrained magnitude, IEEE Trans. Inform. Theory, in press, and in Fix, S. (1977), "Rate Distortion Functions for Continuous Alphabet Memoryless Sources," Ph.D. dissertation, Dept. of Electrical Engineering, Cniversity of Michigan, Aug. 1977.

RrCeIvfed: August 26, 1977; REvisfa: July 21, 1978 


\section{REFERENCES}

Brerger, 'T. (1971), "Rate Distortion Theory," Prentice-Hall, Englewood Cliffs, ․J.

Dobrishin, R. L. (1970), Prescribing a system of random variables by conditional distributions, Theor. Probability Appl. 15, 458-486.

GRAY, R. M., Neuhoff, D. L., AND Shields, P. C. (1975), A generalization of Ornstein's distance with applications to information theory, Ann. Probability 3, 315-328.

I wung, H. M. (1976), Bounds and the evaluation of rate distortion functions, Institute of Statistics Mimeo Series No. 1057, Cniversity of North Carolina at Chapel Hill.

PINSker, M. S. (1964), "Information and Information Stability of Random Variables and Processes," Holden-Day, San Francisco.

TAN, H. H. (1973), "On the Evaluation of Rate Distortion Functions," UCLA School of Engineering and Applied Science Tcch. Rep. No. LCLA-ENG-7356.

TAN, H. H., ANis YAO, K. (1974). Properties of rate-distortion functions. 2. Rate-distortion functions with absolute value distortion measure, in "Source Coding/Ergodic 'Theory Rescarch Seminar" (R. M. Gray, Ed.), pp. 93-101, Stanford University Information Systems Laboratory, 'Гech. Rep. No. 6503-2.

TAN, H. H., AND YAO, K. (1975), Evaluation of rate-distortion functions for a class of independent identically distributed sources under an absolute-magnitude criterion, IEEE Trans. Inform. Theory IT-21, 59-64.

VALLENDER, S. S. (1973), Computing the Wasserstein distance between probability distributions on the line, Theor. Probability Appl. 18, 824-827. 